\title{
Editorial to image processing with soft computing techniques
}

\author{
Irina Perfilieva ${ }^{1} \cdot$ Javier Montero $^{2} \cdot$ Salvatore Sessa $^{3}$
}

Published online: 13 February 2019

(C) Springer-Verlag GmbH Germany, part of Springer Nature 2019

No doubts that image processing is a grateful field for applications of fuzzy techniques. This is due to several factors where the most important one is that a majority of the problems are formulated using vague characterization. The examples are edge detection, where places with "abrupt change" of colors are of interest; segmentation, where the goal is to assign a label to every pixel in an image and by this, make a "partition into multiple segments" possible; sharpening, with the goal to make an image "less blurry" than the original one, etc. As a consequence, fuzzy methods are applied on each step of image processing: from representation and up to evaluation of a quality of a result. In many cases, these methods are even more efficient than the conventional ones.

In this issue, we collected dedicated contributions that propose more than a particular solution to a problem. Our primary concern was about soft computing methods, their technological aspects and proper justification. Moreover, we were aiming at creating a library of methods that can be further used as benchmarks.

The organization of the issue is as follows: we first give papers where new ways of image representation (hyperspectral imaging, image represented by fuzzy function) are proposed; then, we proceed with papers where traditional image processing tasks (edge detection, segmentation, inpainting) are solved using new soft computing methods; finally, some real problems (biometric recognition, meteorological pattern classification, iris localization) where input data given by images are discussed. Below, please

Irina Perfilieva

Irina.Perfilieva@osu.cz

1 Institute for Research and Applications of Fuzzy Modeling, University of Ostrava, 30 dubna 22, 70103 Ostrava 1, Czech Republic

2 Department of Statistics and Operational Research, Complutense University of Madrid, Madrid, Spain

3 Dipartimento di Architettura, Università degli Studi di Napoli Federico II, Naples, Italy find short and relevant description of included contributions.

Hyperspectral imaging using notions from type-2 fuzzy sets, Ainara Lopez-Maestresalas et al.

The paper is focused on a nonstandard image representation and suitable operations of comparison. The proposed representation has a form of a point-to-vector mapping, where each pixel is associated with equally distant wavelengths of a certain range. Despite the familiar vector-form representation, the semantics of components (e.g., strong spatial correlation) are required to develop evolved tools, which better exploit the nature of the data. In this work, the comparison is proposed on the basis of the analogy with type-2 fuzzy sets. The corresponding operators are applied to hyperspectral imaging, and their performance is analyzed in the context of in-lab hyperspectral image segmentation.

Sensitivity analysis for image represented by fuzzy function, Petr Hurtík et al.

The focus is on a new image representation by a fuzzy function instead of pixel-to-color intensity map that is traditionally used for images. It is shown that this representation has certain advantages when the problem of recognitions is considered over distorted images.

A new edge-detection method based on global evaluation using fuzzy clustering, Pablo Arcadio Flores-Vidal et al.

Edge detection is a traditional problem in image processing, the complexity of which depends on the lack of precise definition.

The paper proposes a new strategy to solve this problem using the notion of "edge segment." The latter is specified by a feature vector and then classified as to be (not to be) a good segment. A fuzzy clustering technique is applied for the classification. The approach demonstrates a higher performance compared to other standard edge-detection algorithms. 
An image segmentation technique using nonsubsampled contourlet transform and active contours, Lingling Fang

The problem of image segmentation is attacked by a combination of various methods with the purpose to extract best achievements of each of them.

In some detail, the multiresolution analysis and a particular variational method are applied. The former is exploited to obtain a multiresolution representation, while the latter helps to get the integrity of images. The segmentation results are compared with several state-of-the-art methods.

Total variation with nonlocal FT-Laplacian for patch-based inpainting, Irina Perfilieva et al.

The problem of inpainting (filling in missing or damaged regions) for relatively large damaged areas is considered. Inpainting belongs to the class of inverse problems, so that it does not have a unique solution. The authors selected a variational image denoising model and proposed a modification based on the fuzzy ( $\mathrm{F}-$ ) transform technique. As a result, a new optimization model with nonlocal operators, modified by the F-transform-based operators, is proposed. The minimization algorithm and results are discussed on various examples.

Biometric recognition using finger and palm vein images, Bharathi S. et al.

This contribution is a good example of an application paper, which is focused on both important issues: methodology and realization. The discussed problem is recognition on the basis of biometric input data. In particular, the vascular biometric modalities are considered for authenticating the person. The proposed method provides lower false acceptance rate, false rejection rate and high accuracy of $99.5 \%$ when compared with the existing techniques.
Design of meteorological pattern classification system based on FCM-based radial basis function neural networks using meteorological radar data, Eun-Hu Kim et al.

In this study, a pattern-based classification of meteorological radar data and prediction of heavy rainfalls is realized with the use of a neuro-fuzzy classifier. This problem is of the key importance in Korea where in average per year, a 676.9 billion won of economic loss by rainfall damage is registered. Various combinations of input variables for designing pattern classifier are considered by exploiting the quantitative as well as qualitative characteristic of meteorological radar data. The proposed architecture is designed by using FCM-based radial basis function neural network.

Zadeh max-min composition fuzzy rule for dominated pixel values in iris localization, S. Gino Sophia et al.

The paper aims at solving the Iris localization problem based on the dominated values method which is established based on the Zadeh Max-Min composition. The method may be useful as it provides with a high accuracy, and at the same time, it helps to reduce the search time for localizing the iris. In the comparison with other relevant methods, it visibly shows its potential.

\section{Compliance with ethical standards}

Conflict of interest The author declares that there is no conflict of interest.

Publisher's Note Springer Nature remains neutral with regard to jurisdictional claims in published maps and institutional affiliations. 